\title{
Anatomical location of AICA loop in CPA as a prognostic factor for ISSNHL
}

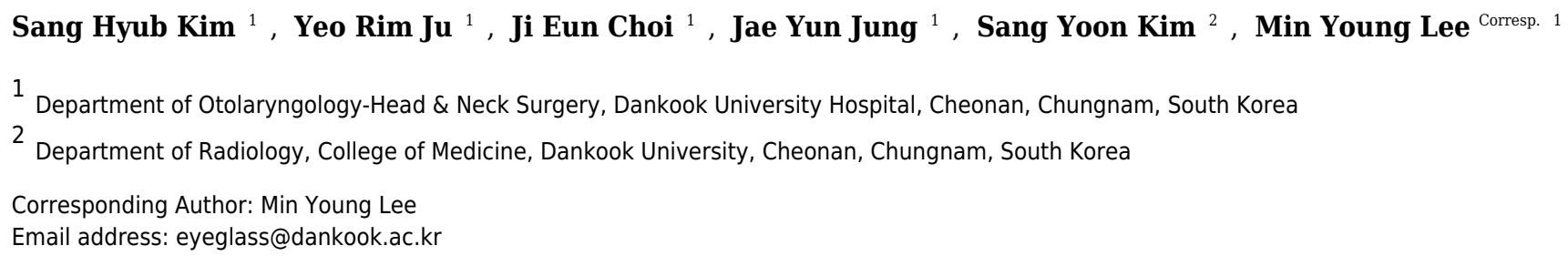

The cerebellopontine angle (CPA) is a triangular-shaped space that lies at the junction of the pons and cerebellum. It contains cranial nerves and the anterior inferior cerebellar artery (AICA). The anatomical shape and location of the AICA is variable within the CPA and internal auditory canal (IAC). A possible etiology of idiopathic sudden sensorineura I hearing loss (ISSNHL) is ischemia of the labyrinthine artery, which is a branch of the AICA. As such, the position of the AICA within the CPA and IAC may be related to the clinical development of ISSNHL. We adopted two methods to classify the anatomic position of the AICA, then analyzed whether these classifications affected the clinical features and prognosis of ISSNHL. We retrospectively reviewed patient data from January 2015 to March 2018. Two established classification methods designed by Cahvada and Gorrie et al. were used. Pure tone threshold at 4 different frequencies $(0.5,1,4$, and $8 \mathrm{kHz})$, at two different time points (at initial presentation and three months after treatment), were analyzed. We compared the affected and unaffected ears, and investigated whether there were any differences in hearing recovery and symptoms between the two classification types. There was no difference in AICA types between ears with and without ISSNHL. Patients who had combined symptoms such as tinnitus and vertigo did not show a different AICA distribution compared with patients who did not. There were differences in quantitative hearing improvement between AICA types, although without statistic significance $(p=0.09-0.13)$. At two frequencies, 1 and $4 \mathrm{kHz}$, there were differences in Chavda types between hearing improvement and no improvement $(p<0.05)$. Anatomical variances of the AICA loop position did not affect the incidence of ISSNHL or co-morbid symptoms including tinnitus and vertigo. In contrast, comparisons of hearing improvement based on Chavda type classification showed a statistical difference, with a higher proportion of Chavda type 1 showing improvements in hearing (AICA outside IAC). 


\section{Anatomical location of AICA loop in CPA as a prognostic factor for ISSNHL}

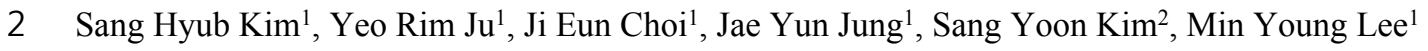

$3{ }^{1}$ Department of Otolaryngology-Head \& Neck Surgery, Dankook University Hospital, South Korea

$4 \quad{ }^{2}$ Department of Radiology, College of Medicine, Dankook University, South Korea

5

6 Corresponding Author: Min Young Lee

7 Department of Otorhinolaryngology-Head \& Neck Surgery, Dankook University Hospital, South Korea

8 E-mail: eyeglass210@gmail.com

9 Tel: $+82-41-550-1785$

FAX: $+82-41-561-3485$

12 Conflict of Interest: None

13 Running title: AICA loop as prognostic factor for SSNHL

14 


\section{Abstract}

17 The cerebellopontine angle (CPA) is a triangular-shaped space that lies at the junction of the pons and cerebellum. It 18 contains cranial nerves and the anterior inferior cerebellar artery (AICA). The anatomical shape and location of the 19 AICA is variable within the CPA and internal auditory canal (IAC). A possible etiology of idiopathic sudden 20 sensorineural hearing loss (ISSNHL) is ischemia of the labyrinthine artery, which is a branch of the AICA. As such, 21 the position of the AICA within the CPA and IAC may be related to the clinical development of ISSNHL.

22 We adopted two methods to classify the anatomic position of the AICA, then analyzed whether these classifications 23 affected the clinical features and prognosis of ISSNHL.

We retrospectively reviewed patient data from January 2015 to March 2018. Two established classification methods designed by Chavda and Gorrie et al. were used. Pure tone threshold at 4 different frequencies $(0.5,1,4$, and $8 \mathrm{kHz})$, at two different time points (at initial presentation and three months after treatment), were analyzed. We compared the affected and unaffected ears, and investigated whether there were any differences in hearing recovery and symptoms between the two classification types.

There was no difference in AICA types between ears with and without ISSNHL. Patients who had combined symptoms such as tinnitus and vertigo did not show a different AICA distribution compared with patients who did not. There were differences in quantitative hearing improvement between AICA types, although without statistical significance ( $\mathrm{p}=0.09-0.13$ ). At two frequencies, 1 and $4 \mathrm{kHz}$, there were differences in Chavda types between hearing improvement and no improvement $(\mathrm{p}<0.05)$. 
40

41 The cerebellopontine angle (CPA) is triangular-shaped space filled with cerebrospinal fluid, and is located at the

42 junction of the pons and cerebellum. It contains several crucial structures such as cranial nerves V to VIII, and

43 arteries such as superior cerebellar artery (SCA) and anterior inferior cerebellar artery (AICA). The internal auditory canal (IAC), which is a nerve canal surrounded by bone, rises anterolaterally from the CPA to reach the peripheral cochleovestibular organs. The IAC contains cranial nerves VII and VIII, which are ultimately responsible for facial muscle movement, hearing, and balance.(Rhoton 2000)

The AICA is a branch of the basilar artery and courses through the CPA posterolaterally to supply the anterior to middle parts of the cerebellum and inferolateral pons. It branches into the labyrinthine artery, which is the sole vascular supply for the labyrinth, cochlea, and vestibular organs. The anatomical shape and location of the AICA is variable in the CPA. (Kim et al. 1990) In postmortem and imaging studies, it has been found within the IAC in 15 to 40\% of patients. (De Carpentier et al. 1996; Mazzoni \& Hansen 1970; Reisser \& Schuknecht 1991)

Idiopathic sudden sensorineural hearing loss (ISSNHL) is a commonly seen disease in the otologic clinic. However, there is no known pathophysiology and current treatment relies on the use of systemic or intra-tympanic steroids. Possible hypotheses include inflammation, labyrinthine artery occlusion, or damage to the cochlear nerve.(Byl Jr 1984; Merchant et al. 2005) Given that the labyrinthine artery is a branch of the AICA, it is plausible that differences in the anatomical variation of the AICA results in the clinical findings of ISSNHL.

A number of studies have shown cases of hearing loss with an AICA located within the IAC, (Moosa et al. 2015) and have correlated various AICA locations with audio-vestibular symptoms (Chadha \& Weiner 2008; De Carpentier et al. 1996; Gorrie et al. 2010; Kazawa et al. 2013). However, none of these studies has focused on ISSNHL, which could have a different pathophysiology given the vast differences in clinical features and diagnostic criteria. As such, in the present study, we used two previously reported methods of classification to reveal the correlation of distance of AICA loop and IAC (Chavda type) or contact of nerves and vessel (Gorrie type) to hearing status. We then analyzed whether these classifications affected the clinical features and prognosis of ISSNHL. 


\section{Materials and Methods}

66

67

68

\section{Subjects and design}

We retrospectively reviewed patient data from January 2015 to March 2018. This study was approved by the institutional review board of Dankook University Hospital (Ethical Application Ref: 2017-08-003). All patients who were admitted for ISSNHL were enrolled in the study. Verbal informed consents from participants were received. ISSNHL was diagnosed according to traditional criteria, which is defined as a threshold shift of greater than $30 \mathrm{~dB}$ in three consecutive frequencies, or if the patient has new hearing loss in a duration less than 3 days. (Anderson $\&$ Meyerhoff 1983; Mattox \& Simmons 1977; Schuknecht \& Donovan 1986) Demographic data are shown in Table 1. The vestibular involvement was relatively higher in our experimental group compared to previous reports (Moskowitz et al. 1984; Park et al. 2001; Shaia \& Sheehy 1976). This could be related to subjects who were enrolled in this study, since the MRI is not a routine study for hearing loss in our health system. Patients with MRI imaging of the IAC with no signs of vestibular schwannoma were included in the study. Patients were classified according to the anatomical location of the AICA and cranial nerves within the IAC. Two established classification methods designed by Chavda(McDermott et al. 2003) and Gorrie(Gorrie et al. 2010) et al. were used. Pure tone threshold at four different frequencies $(0.5,1,4$, and $8 \mathrm{kHz})$, at two different time points (at time of initial presentation, and three months after initial treatment), were analyzed. All patients were given anti-viral agent, systemic high dose steroid therapy (48mg at day time, $12 \mathrm{mg}$ at night time, total $60 \mathrm{mg}$ of methylprednisolone for 7 days) and non-systemic steroid responsive (mean recovery average less than $10 \mathrm{~dB}$ HL) subjects had additional intra-tympanic steroid injections. We compared the affected and unaffected ears with two different classification systems, and investigated whether there were differences in hearing recovery and symptoms. Hearing improvement was assessed by Siegel's criteria (average of 0.5, 1, 2 and 4kHz)(Siegel 1975) and measuring the first threshold shift between time points at each frequency, and documenting the proportion of patients with improved hearing $(>10 \mathrm{~dB} \mathrm{HL})$ at each separate frequency.

\section{MRI protocol}

All MRI test were conducted using a 3 T scanner (signa HDxt, GE Medical system, Milwaukee, WI) with 8 channel head coil. Among the routine IAC MR imaging protocol, 3D T2 VISTA images were selected for analyzing the anatomical configurations of IAC vessel and cranial nerves. Two classification systems were adopted. The first was the Chavda classification published by McDermott et al. (McDermott et al. 2003). This system classifies AICA types as follows: type 1 is an AICA loop within the CPA but outside the IAC; type 2 is an AICA loop extending into the IAC but is less than $50 \%$ the length of the IAC; type 3 is an AICA loop with greater than $50 \%$ extension into the IAC (Fig. 1). The second classification system used was the Gorrie type, which is based on the amount of contact of between the AICA and adjacent cranial nerves. Type 1 is an AICA loop without contact to adjacent nerves; type 2 is an AICA loop that runs adjacent to the nerves; type 3 is an AICA loop that physically displaces the $8^{\text {th }}$ cranial nerve; type 4 is an AICA loop that courses between the $7^{\text {th }}$ and $8^{\text {th }}$ cranial nerves (Fig. 2). All MR images were analyzed and classified by a radiologist who is co-author of our manuscript (SYK). 


\section{Statistical analysis}

101 All data were analyzed by GraphPad Prism (GraphPad Software, La Jolla, CA, USA) or SPSS (IBM SPSS statistics,

102 Armonk, NY, USA) software. A Shapiro-Wilk normality test was used to determine whether the data were

103 parametric or non-parametric. Significant differences between groups were statistically analyzed using t-test in cases

104 of a parametric distribution, and Mann-Whitney U test in cases of a nonparametric distribution. Fischer's exact test

105 was used for the cross-table analysis. A p-value less than 0.05 was considered statistically significant.

106

107 
109

110

111

112

113

114

115

116

117

118

119

120

121

122

123

124

125

126

127

128

129

130

131

132

133

134

135

136

137

138

139

\section{Results}

Pure tone averages of ears with ISSNHL were 73.6, 76.9, 78.1, and 77.1 at $0.5,1,4$ and $8 \mathrm{kHz}$ respectively, and those of the contralateral side were 11.5, 12.3, 23.3, and 29.7 at $0.5,1,4$ and $8 \mathrm{kHz}$ respectively. The average threshold shift of the contralateral ear at each frequency was no greater than $30 \mathrm{~dB}$ HL, suggesting near normal hearing function. We compared the anatomical position of the AICA loop between ears with ISSNHL and the unaffected contralateral ear. The types of anatomical variations of the AICA were not different between the affected side and the contralateral side (Table 2) ( $p>0.05$, Fisher's exact test). With regard to the Chavda classification, Chavda type I was the most common, followed by type II, and type III. For the Gorrie classification, the Gorrie type III was the most common (Table 2).

We also analyzed symptoms such as vertigo and tinnitus. The relationship between AICA and symptoms were classified as Table 3 and 4. As a result, the anatomic variations of AICA was not different according to vertigo and tinnitus, respectively $(\mathrm{p}>0.05)$.

We compared the threshold shift from the start of the treatment and at 3 months. In all four frequencies, Chavda type 1 showed the largest threshold improvement but was not statistically significant (Fig. 3) (500Hz: Kruskal-Wallis test, $\mathrm{KW}$ statistics $=4.091, \mathrm{p}=0.13$; $1 \mathrm{kHz}$ : Kruskal-Wallis test, $\mathrm{KW}$ statistics $=4.719, \mathrm{p}=0.09 ;$ 4kHz: Kruskal-Wallis test, $\mathrm{KW}$ statistics $=4.789, \mathrm{p}=0.09$; 8kHz: Kruskal-Wallis test, KW statistics 3.336, $\mathrm{p}=0.19$, mean hearing level: KruskalWallis test, $\mathrm{KW}$ statistics $3.381, \mathrm{p}=0.18)$. At lower frequencies $(500 \mathrm{~Hz}, 1 \mathrm{kHz})$, hearing improvements were found in type 2 and type 3 Gorrie configurations. Higher frequencies $(4 \mathrm{kHz}, 8 \mathrm{kHz})$ did not yield any significant differences in hearing improvements, with a Gorrie type 4 at $4 \mathrm{kHz}$ improving the least. These differences were not statistically significant (Fig. 4) (500Hz: Kruskal-Wallis test, KW statistics=2.770, p=0.43; 1kHz: Kruskal-Wallis test, KW statistics $=3.811, \mathrm{p}=0.28$; $4 \mathrm{kHz}$ : Kruskal-Wallis test, $\mathrm{KW}$ statistics $=3.609, \mathrm{p}=0.31 ; 8 \mathrm{kHz}$ : Kruskal-Wallis test, $\mathrm{KW}$ statistics $0.2900, \mathrm{p}=0.96$, mean hearing level: Kruskal-Wallis test, $\mathrm{KW}$ statistics $3.277, \mathrm{p}=0.35$ ). According to the classification of Siegel's criteria, it was found that improved groups, from slight to complete recovery, showed higher Chavda type 1 proportion ( $>50 \%$ ) compared to no recovery (Chavda type $1<30 \%$ ) but it failed to reveal statistical significance (Fischer exact test, $\mathrm{p}=0.50$ ). As hearing improved across both classifications, all Chavda types had a significant difference at $1 \mathrm{kHz}$ and $4 \mathrm{kHz}$ (Fischer exact test, $1 \mathrm{kHz}$ : $\mathrm{p}=0.03,4 \mathrm{kHz}$ : $\mathrm{p}=0.01$ ). There was no statistical significance at other frequencies in Chavda or Gorrie type configurations (Chavda type; Fischer exact test, $500 \mathrm{~Hz}: \mathrm{p}=0.17,8 \mathrm{kHz}$ : $\mathrm{p}=0.14$ ) (Gorrie type; Fischer exact test, $500 \mathrm{~Hz}$ : $\mathrm{p}=0.47,1 \mathrm{kHz}$ : $\mathrm{p}=0.36,4 \mathrm{kHz}$ : $\mathrm{p}=0.11,8 \mathrm{kHz}$ : $\mathrm{p}=0.92)($ Table 5 and 6 ). 


\section{Discussion}

142 In the present study, there were no differences in AICA types in ears with or without ISSNHL. Patients who had 143 combined symptoms such as tinnitus and vertigo did not show a different distribution of AICA type compared to 144 patients without symptoms. This suggests that AICA type does not affect the incidence of ISSNHL and concurrent 145 symptoms. There were some differences in quantitative hearing improvement between types, although without 146 statistical significance ( $\mathrm{p}$ values between 0.09 and 0.13 ). At two frequencies, 1 and $4 \mathrm{kHz}$, there was a difference in

147 Chavda types between patients who experienced hearing improvement and patients who did not. In groups that had 148 improvements in hearing, we found a higher proportion of Chavda type 1 configurations (AICA locating outside the 149 IAC). These results suggest that the anatomic location of the AICA loop may help prognosticate hearing outcomes 150 in ISSNHL patients.

151 Currently, there is no clear etiology of AICA loop formation and anatomical variances seen in AICA positions.

152 Hypotheses include senile elongation of the artery, arteriosclerosis, and arachnoid adhesions between nerves and 153 vessels.(Applebaum \& Valvassori 1984) The prevalence of AICA loops inside the IAC is thought to be 154 approximately 13 to $40 \%$ in cadaveric dissections (Mazzoni \& Hansen 1970; Reisser \& Schuknecht 1991) and 14 to 155 34\% in imaging studies using MRI. (De Carpentier et al. 1996; Sirikci et al. 2005) In the current study the 156 percentage of patients found to have a Chavda type 1 configuration was $51 \%$ ( $59 \%$ in control side), which is a 157 smaller than results from previous studies (60 to 87\%). This difference may be attributable to variations in study 158 number, age, or sex. Future studies that match healthy controls with subjects may be useful in further evaluating the 159 relationship between ISSNHL incidence and AICA loop location.

160 Microvascular compression is thought to be responsible for certain cases of hearing loss, tinnitus, vertigo, and 161 hemifacial spasm.(Jannetta 1980) The results of our study are in line with previous findings, with no differences 162 being found in the relationship between AICA loop distribution and symptomatic/non-symptomatic patients.(De 163 Carpentier et al. 1996; Gorrie et al. 2010; Sirikci et al. 2005) We also did not find a higher incidence of Gorrie type 1643 and 4 configurations in patients with tinnitus, thereby decreasing the likelihood that symptoms could be due to 165 contact of the AICA loop with the cochlear nerve. However, given the low percentage of pulsatile tinnitus compared 166 to subjective tinnitus patients in our study, we believe that our current data are insufficient to comment further on 167 the previously studied (Chadha \& Weiner 2008; De Ridder et al. 2005) relationship between tinnitus (vascular and 168 non-vascular) and AICA loop position in ISSNHL patients.

169 Quantitative hearing improvement failed to reveal significant differences, although the patient group that had 170 hearing improvement showed different Chavda type proportions. This finding may be due to chance or to low 171 sample sizes. Nevertheless, a plausible explanation for the improved prognosis of Chavda type 1 configurations is 172 necessary. Among many possible etiologies of ISSNHL, two most highly adopted theories are viral inflammation 173 and ischemia. Inflammation of cochlear nerve can be due to a variety of infectious causes, and results in reversible 174 axonal swelling and degeneration. Most cases retain a good prognosis with appropriate therapeutic management 175 with appropriate management such as steroid (to reduced secondary damage due to edema) and antiviral agent. In 
176

177

178

179

180

181

182

183

184

185

186

187

188

189

190

191

192

193

194

195

196

197

198

199

200

201

202

203

204

contrast, ischemic attack results in irreversible cochlear damage due to sensorineural cell death, (Izumikawa et al. 2005) leading to poorer outcomes. Given that the pathophysiology of ISSNL is thought to be multifactorial, the group that experienced less hearing improvements (Chavda type 2 or 3) may have had a higher rate of vascular etiologies compared to patients with a Chavda type 1 configuration. Unlike Gorrie classification which is classified by the contact of vessel and nerves, Chavda classification divides groups by distance of AICA loop and IAC. In case of Chavda type 2 and 3, AICA loop is located within the narrow IAC which in many case leads to smaller diameter of AICA loop and sudden rotation. On the contrary, in the Chavda type 1, AICA loop is formed in CPA outside of IAC which has relatively larger space, abrupt rotation of the loop is not always necessary in this case. The turbulence, which is relevant factor in thrombus formation within the AICA loop (Bluestein et al. 1997; Deusebio et al. 2014), may occur at a higher rate in Chavda type 2 or 3 patients (small diameter IAC loop and narrow space), and supports the notion that labyrinthine artery ischemia is more common in these patients, resulting in a higher rate of no-improvement hearing outcome from ISSNHL. Furthermore, outcome variabilities among different frequencies are another evidence to speculate the pathophysiology. The statistical group difference was observed in $1,4 \mathrm{kHz}$ not in $500 \mathrm{~Hz}$ and $8 \mathrm{kHz}$. The possible reason for no group difference in $8 \mathrm{kHz}$ might be related to small hearing improvements, as observed in Figure 3 and 4 . On the other hand, $500 \mathrm{~Hz}$ hearing improvement was relatively similar to other frequencies (Fig. 3 and 4) and there should be an alternate plausible theory. Considering the tonotopicity of cochlear nerve(Muller 1991), the frequencies which showed group differences (better improvement in Chavda type 1) would be the peripheral part of cochlear nerve (except the highest frequency). In treatment responsive population which had high proportion of Chavda type 1 (could be viral origin); damage of cochlear nerve fiber could be focused in peripheral axons which are close to the nerve sheath and susceptible to the pressure increase.

On the other hand, it is possible to argue that in Chavda type 1 response to treatment was better compared to other types. Systemically delivered steroid and antiviral agent could reach the target organ faster in case of lesser complicated anatomical positioning of AICA, such as Chavda type 1, studies comparing outcomes and AICA classifications of the local and systemic treatment would help better understanding considering this point.

\section{Conclusion}

Anatomical variances in AICA loop position did not affect the incidence of ISSNHL or co-morbid symptoms. In contrast, comparisons between groups with improvements in hearing and those without revealed that a higher proportion of Chavda type 1 (AICA outside IAC) patients had better prognostic outcomes. 


\section{Reference}

Anderson RG, and Meyerhoff WL. 1983. Sudden sensorineural hearing loss. Otolaryngol Clin North Am 16:189195.

Applebaum EL, and Valvassori GE. 1984. Auditory and vestibular system findings in patients with vascular loops in the internal auditory canal. Ann Otol Rhinol Laryngol Suppl 112:63-70.

Bluestein D, Niu L, Schoephoerster RT, and Dewanjee MK. 1997. Fluid mechanics of arterial stenosis: relationship to the development of mural thrombus. Ann Biomed Eng 25:344-356.

Byl Jr FM. 1984. Sudden hearing loss: eight years' experience and suggested prognostic table. Laryngoscope 94:647-661.

Chadha NK, and Weiner GM. 2008. Vascular loops causing otological symptoms: a systematic review and metaanalysis. Clin Otolaryngol 33:5-11. 10.1111/j.1749-4486.2007.01597.x

De Carpentier J, Lynch N, Fisher A, Hughes D, and Willatt D. 1996. MR imaged neurovascular relationships at the cerebellopontine angle. Clin Otolaryngol Allied Sci 21:312-316.

De Ridder D, De Ridder L, Nowe V, Thierens H, Van de Heyning P, and Moller A. 2005. Pulsatile tinnitus and the intrameatal vascular loop: why do we not hear our carotids? Neurosurgery 57:1213-1217.

Deusebio E, Boffetta G, Lindborg E, and Musacchio S. 2014. Dimensional transition in rotating turbulence. Phys Rev E Stat Nonlin Soft Matter Phys 90:023005. 10.1103/PhysRevE.90.023005

Gorrie A, Warren FM, 3rd, de la Garza AN, Shelton C, and Wiggins RH, 3rd. 2010. Is there a correlation between vascular loops in the cerebellopontine angle and unexplained unilateral hearing loss? Otol Neurotol 31:4852.

Izumikawa M, Minoda R, Kawamoto K, Abrashkin KA, Swiderski DL, Dolan DF, Brough DE, and Raphael Y. 2005. Auditory hair cell replacement and hearing improvement by Atoh1 gene therapy in deaf mammals. Nat Med 11:271-276.

Jannetta PJ. 1980. Neurovascular compression in cranial nerve and systemic disease. Ann Surg 192:518-525.

Kazawa N, Togashi K, and Ito J. 2013. The anatomical classification of AICA/PICA branching and configurations in the cerebellopontine angle area on 3D-drive thin slice T2WI MRI. Clin Imaging 37:865-870. 10.1016/j.clinimag.2011.11.021

Kim HN, Kim YH, Park IY, Kim GR, and Chung IH. 1990. Variability of the surgical anatomy of the neurovascular complex of the cerebellopontine angle. Ann Otol Rhinol Laryngol 99:288-296. $10.1177 / 000348949009900408$

Mattox DE, and Simmons FB. 1977. Natural history of sudden sensorineural hearing loss. Ann Otol Rhinol Laryngol $86: 463-480$.

Mazzoni A, and Hansen CC. 1970. Surgical anatomy of the arteries of the internal auditory canal. Arch Otolaryngol 91:128-135.

McDermott AL, Dutt SN, Irving RM, Pahor AL, and Chavda SV. 2003. Anterior inferior cerebellar artery syndrome: fact or fiction. Clin Otolaryngol Allied Sci 28:75-80.

Merchant SN, Adams JC, and Nadol Jr JB. 2005. Pathology and pathophysiology of idiopathic sudden sensorineural hearing loss. Otology \& Neurotology 26:151-160.

Moosa S, Fezeu F, Kesser BW, Ramesh A, and Sheehan JP. 2015. Sudden unilateral hearing loss and vascular loop in the internal auditory canal: case report and review of literature. J Radiosurg SBRT 3:247-255.

Moskowitz D, Lee KJ, and Smith HW. 1984. Steroid use in idiopathic sudden sensorineural hearing loss. Laryngoscope 94:664-666.

Muller M. 1991. Frequency representation in the rat cochlea. Hear Res 51:247-254.

Park HM, Jung SW, and Rhee CK. 2001. Vestibular diagnosis as prognostic indicator in sudden hearing loss with vertigo. Acta Otolaryngol Suppl 545:80-83.

Reisser C, and Schuknecht HF. 1991. The anterior inferior cerebellar artery in the internal auditory canal. Laryngoscope 101:761-766. 10.1288/00005537-199107000-00012

Rhoton AL, Jr. 2000. The cerebellopontine angle and posterior fossa cranial nerves by the retrosigmoid approach. Neurosurgery 47:S93-129.

Schuknecht HF, and Donovan ED. 1986. The pathology of idiopathic sudden sensorineural hearing loss. Arch Otorhinolaryngol 243:1-15.

Shaia FT, and Sheehy JL. 1976. Sudden sensori-neural hearing impairment: a report of 1,220 cases. Laryngoscope 86:389-398. 10.1288/00005537-197603000-00008

Siegel LG. 1975. The treatment of idiopathic sudden sensorineural hearing loss. Otolaryngol Clin North Am 8:467- 
260

261

262

263

264

265

266

267

268

269

270

271

272

273

274

275

276

277

278

279

280

281

282

283

284

285

286

287

288

289

290

291

292

473.

Sirikci A, Bayazit Y, Ozer E, Ozkur A, Adaletli I, Cuce MA, and Bayram M. 2005. Magnetic resonance imaging based classification of anatomic relationship between the cochleovestibular nerve and anterior inferior cerebellar artery in patients with non-specific neuro-otologic symptoms. Surg Radiol Anat 27:531-535.

\section{Figure legends}

\section{Figure 1. Chavda classification of AICA loop}

(A) is showing AICA loop (arrow) observed in cerebellopontine angle (CPA) outside the internal auditory canal (IAC) which is type 1. (B) is showing type 2 in which AICA loop (arrow) is occupying no more than $50 \%$ of IAC. (C) is type 3 that AICA loop (arrow) leaches more than $50 \%$ of total length of IAC.

\section{Figure 2. Gorrie classification of AICA loop}

(A) is showing AICA loop (arrow) running separate from cranial nerve which is type 1. (B) is showing type 2 in which AICA loop (arrow) is running adjacent cranial nerve. (C) is type 3 that AICA loop (arrow) deflects the $8^{\text {th }}$ cranial neve and (D) is type 4 that AICA loop (arrow) runs between $7^{\text {th }}$ and $8^{\text {th }}$ cranial nerve.

\section{Figure 3. Hearing threshold improvement across different Chavda types}

At all frequencies (A-D), highest threshold improvements were observed in Chavda type 1. At lowest frequency $(500 \mathrm{~Hz},(\mathrm{~A}))$, similar hearing improvement were observed in Chavda type 2 and 3 . At $1 \mathrm{kHz}$, Chavda type 3 showed higher improvement (B). In contrast, both in $4 \mathrm{kHz}$ (C) and $8 \mathrm{kHz}$ (D), Chavda type 2 showed higher improvement. Average of 0.5, 1, 2 and $4 \mathrm{kHz}$ was compared and revealed high improvement in Chavda type 1 (E). In Siegel's criteria (F), proportion of Chavda type 1 was small in no improvement group. Nevertheless, all of these comparisons among Chavda types failed to reveal statistical significance (see the results for detailed statistics). The number in center of each bar means each mean hearing threshold improvement (dB HL). Error bar indicates standard deviation.

\section{Figure 4. Hearing threshold improvement across different Gorrie types}

At $500 \mathrm{~Hz}$ (A) and $1 \mathrm{kHz}(\mathrm{B})$, Gorrie type 2 and 3 showed higher threshold improvement. At $4 \mathrm{kHz}$ (C) Gorrie type 4 showed least improvement of hearing. At $8 \mathrm{kHz}(\mathrm{D})$, threshold improvement across Gorrie types were similar. Average of $0.5,1,2$ and $4 \mathrm{kHz}$ was compared and showed similar Gorrie type distribution to $500 \mathrm{~Hz}$ and $1 \mathrm{kHz}(\mathrm{E})$. In Siegel's criteria (F), proportion of Gorrie types was not different among groups. Nevertheless, all of these comparisons among Gorrie types failed to reveal statistical significance (see the results for detailed statistics). The number in center of each bar means each mean hearing threshold improvement (dB HL). Error bar indicates standard deviation. 


\section{Table $\mathbf{1}$ (on next page)}

Demographic data of patients in present study 
2

3

4

5

6

7

8

9

10

11

12

13

14

15

16

\begin{tabular}{|c|c|}
\hline Demographics & $45.0( \pm 15.3)$ \\
\hline $\begin{array}{c}\text { Mean age } \\
( \pm \text { Standard deviation })\end{array}$ & $3.7( \pm 2.2)$ \\
\hline $\begin{array}{c}\text { Hospital day of MR imaging } \\
( \pm \text { Standard deviation })\end{array}$ & M 59\% vs F $41 \%$ \\
\hline Gender &
\end{tabular}

\begin{tabular}{|c|c|}
\hline $\begin{array}{c}\text { Patients with } \\
\text { Accompanying symptom }\end{array}$ & \\
\hline Vertigo (\%) & $31(63.3)$ \\
\hline Tinnitus (\%) & $31(63.3)$ \\
\hline
\end{tabular}

Table 1. Demographic data of patients in present study 


\section{Table 2 (on next page)}

Chavda type and Gorrie type distributions in ISSNHL and contralateral ear 


\begin{tabular}{|l|c|c|c|}
\hline & $\begin{array}{c}\text { Ipsilateral ear } \\
(\%)\end{array}$ & $\begin{array}{c}\text { Contralateral ear } \\
(\%)\end{array}$ & \multirow{2}{*}{0.651} \\
\cline { 1 - 3 } Chavda type I & $25(51.0 \%)$ & $29(59.2 \%)$ & \\
\cline { 1 - 3 } Chavda type II & $22(44.9 \%)$ & $19(38.8 \%)$ & \multirow{2}{*}{0.598} \\
\cline { 1 - 3 } Chavda type III & $2(4.1 \%)$ & $1(2.0 \%)$ & \multirow{2}{*}{} \\
\cline { 1 - 3 } Gorrie type I & $7(14.3 \%)$ & $8(16.3 \%)$ & \\
\cline { 1 - 3 } Gorrie type II & $9(18.4 \%)$ & $24(49.0 \%)$ & \\
\cline { 1 - 3 } Gorrie type III & $29(59.2 \%)$ & $7(14.3 \%)$ & \\
\cline { 1 - 3 } Gorrie type IV & $4(8.1 \%)$ & &
\end{tabular}

1

Table 2. Chavda type and Gorrie type distributions in ISSNHL and contralateral ear

3

4 


\section{Table 3(on next page)}

Chavda type and Gorrie type distributions in ISSNHL with vertigo and without vertigo 


\begin{tabular}{|l|c|c|c|}
\hline & $\begin{array}{c}\text { With vertigo } \\
(\%)\end{array}$ & $\begin{array}{c}\text { Without vertigo } \\
(\%)\end{array}$ & \multirow{2}{*}{0.528} \\
\cline { 1 - 3 } Chavda type I & $15(48.4 \%)$ & $10(55.6 \%)$ & \\
\cline { 1 - 3 } Chavda type II & $14(45.2 \%)$ & $8(44.4 \%)$ & \multirow{2}{*}{0.861} \\
\cline { 1 - 3 } Chavda type III & $2(6.4 \%)$ & $0(0.0 \%)$ & \\
\hline Gorrie type I & $5(16.1 \%)$ & $2(11.1 \%)$ & \\
\cline { 1 - 3 } Gorrie type II & $6(19.4 \%)$ & $12(16.7 \%)$ & \\
\hline Gorrie type III & $17(54.8 \%)$ & $1(5.5 \%)$ & \\
\cline { 1 - 3 } Gorrie type IV & $3(9.7 \%)$ & &
\end{tabular}

2 Table 3. Chavda type and Gorrie type distributions in ISSNHL with vertigo and without vertigo 3 


\section{Table 4(on next page)}

Chavda type and Gorrie type distributions in ISSNHL with and without tinnitus 


\begin{tabular}{|l|c|c|c|}
\hline & $\begin{array}{c}\text { With tinnitus } \\
(\%)\end{array}$ & $\begin{array}{c}\text { Without tinnitus } \\
(\%)\end{array}$ & p-value \\
\cline { 1 - 3 } Chavda type I & $21(55.3 \%)$ & $4(36.4 \%)$ & \multirow{2}{*}{0.414} \\
\cline { 1 - 3 } Chavda type II & $16(42.1 \%)$ & $6(54.5 \%)$ & \\
\cline { 1 - 3 } Chavda type III & $1(2.6 \%)$ & $1(9.1 \%)$ & \multirow{2}{*}{0.208} \\
\hline Gorrie type I & $5(13.2 \%)$ & $2(18.2 \%)$ & \\
\cline { 1 - 3 } Gorrie type II & $9(23.7 \%)$ & $0(0.0 \%)$ & \\
\cline { 1 - 3 } Gorrie type III & $22(57.9 \%)$ & $2(63.6 \%)$ & \\
\cline { 1 - 3 } Gorrie type IV & $2(5.2 \%)$ & $2(18.2 \%)$ & \\
\hline
\end{tabular}

1

Table 4. Chavda type and Gorrie type distributions in ISSNHL with and without tinnitus

3

4

5 
Table 5 (on next page)

Chavda type proportion of hearing improved cases at each frequencies 
1

\begin{tabular}{|l|c|c|c|c|}
\hline & $\begin{array}{c}\text { Cahvada type I } \\
(\mathrm{n}=25)\end{array}$ & $\begin{array}{c}\text { Cahvada type 2 } \\
(\mathrm{n}=22)\end{array}$ & $\begin{array}{c}\text { Cahvada type 3 } \\
(\mathrm{n}=2)\end{array}$ & $p$-value \\
\hline $500 \mathrm{~Hz}(\%)$ & $17(68.0 \%)$ & $9(40.9 \%)$ & $1(50.0 \%)$ & 0.174 \\
\hline $\mathbf{K H z}(\%)$ & $\mathbf{1 9 ( 7 6 . 0 \% )}$ & $\mathbf{9 ( 4 0 . 9 \% )}$ & $\mathbf{1 ( 5 0 . 0 \% )}$ & $\mathbf{0 . 0 4 9}$ \\
\hline $\mathbf{4 K H z}(\%)$ & $\mathbf{1 9 ( 7 6 . 0 \% )}$ & $\mathbf{9 ( 4 0 . 9 \% )}$ & $\mathbf{0 ( 0 . 0 \% )}$ & $\mathbf{0 . 0 1 3}$ \\
\hline $8 \mathrm{KHz}(\%)$ & $13(52.0 \%)$ & $6(27.2 \%)$ & $0(0.0 \%)$ & 0.114 \\
\hline
\end{tabular}

2 Bold: statistically significant

3

4 Table 5. Chavda type proportion of hearing improved cases at each frequencies 


\section{Table 6 (on next page)}

Gorrie type proportion of hearing improved cases at each frequencies 


\begin{tabular}{|l|c|c|c|c|c|}
\hline & $\begin{array}{c}\text { Gorrie type 1 } \\
(\mathrm{n}=7)\end{array}$ & $\begin{array}{c}\text { Gorrie type 2 } \\
(\mathrm{n}=9)\end{array}$ & $\begin{array}{c}\text { Gorrie type 3 } \\
(\mathrm{n}=29)\end{array}$ & $\begin{array}{c}\text { Gorrie type 4 } \\
(\mathrm{n}=4)\end{array}$ & $p$-value \\
\hline $500 \mathrm{~Hz}(\%)$ & $3(42.8 \%)$ & $6(66.6 \%)$ & $17(58.6 \%)$ & $1(25.0 \%)$ & 0.472 \\
\hline $1 \mathrm{KHz}(\%)$ & $4(57.2 \%)$ & $7(77.7 \%)$ & $17(58.6 \%)$ & $1(25.0 \%)$ & 0.356 \\
\hline $4 \mathrm{KHz}(\%)$ & $4(57.2 \%)$ & $6(66.6 \%)$ & $18(62.0 \%)$ & $0(0.0 \%)$ & 0.114 \\
\hline $8 \mathrm{KHz}(\%)$ & $3(42.8 \%)$ & $4(44.4 \%)$ & $11(37.9 \%)$ & $1(25.0 \%)$ & 0.919 \\
\hline
\end{tabular}

1

2 Table 6. Gorrie type proportion of hearing improved cases at each frequencies

3 


\section{Figure 1}

\section{Chavda classification of AICA loop}

(A) is showing AICA loop (arrow) observed in cerbellopontine angle (CPA) outside the internal auditory canal (IAC) which is type 1. (B) is showing type 2 in which AICA loop (arrow) is occupying no more than $50 \%$ of IAC. (C) is type 3 that AICA loop (arrow) leaches more than $50 \%$ of total length of IAC.

*Note: Auto Gamma Correction was used for the image. This only affects the reviewing manuscript. See original source image if needed for review.
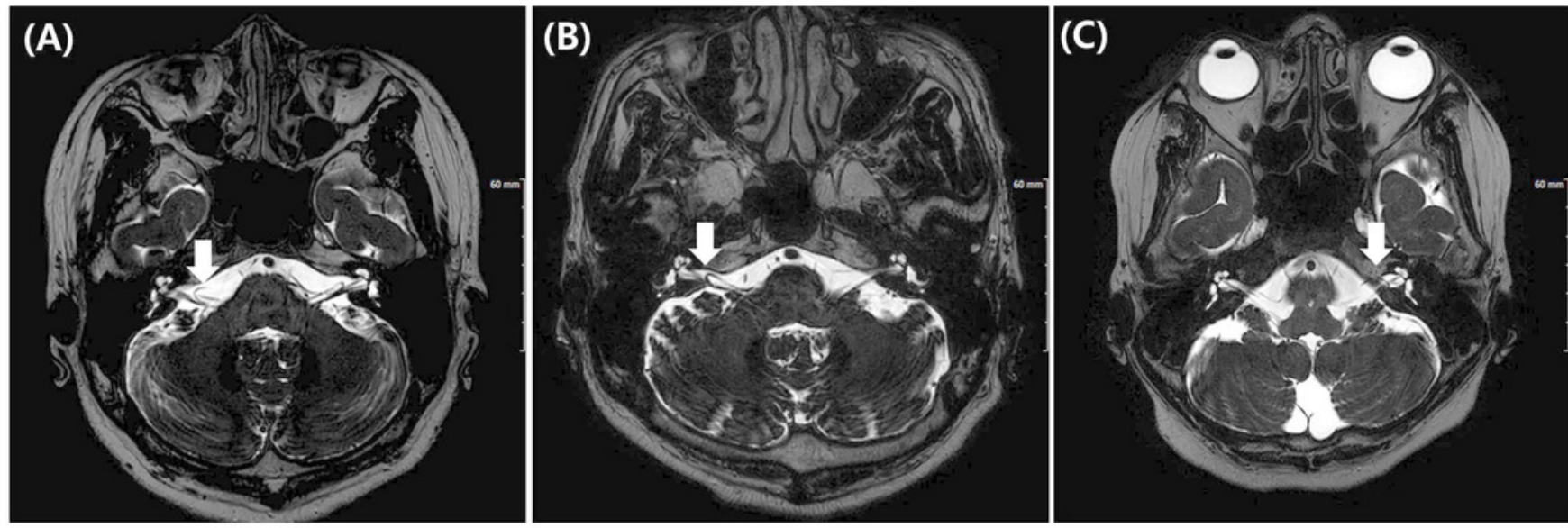


\section{Figure 2}

Gorrie classification of AICA loop

(A) is showing AICA loop (arrow) running separate from cranial nerve which is type $1 .(B)$ is showing type 2 in which AICA loop (arrow) is running adjacent cranial nerve. (C) is type 3 that AICA loop (arrow) deflects the $8^{\text {th }}$ cranial neve and (D) is type 4 that AICA loop (arrow) runs between $7^{\text {th }}$ and $8^{\text {th }}$ cranial nerve.
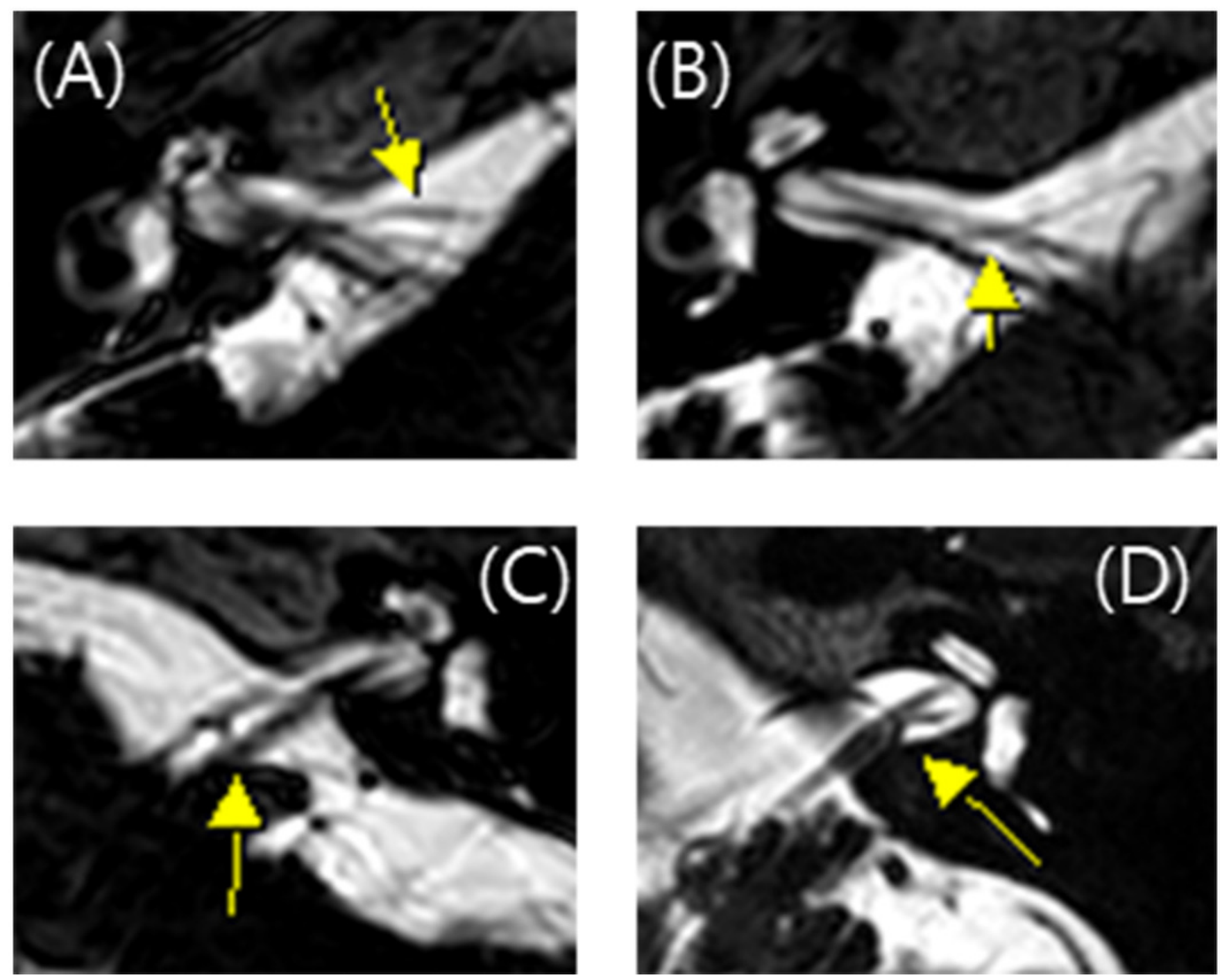


\section{Figure 3}

Hearing threshold improvement across different Chavda types

At all frequencies (A-D), highest threshold improvements were observed in Chavda type 1. At lowest frequency $(500 \mathrm{~Hz},(\mathrm{~A}))$, similar hearing improvement were observed in Chavda type 2 and 3. At $1 \mathrm{kHz}$, Chavda type 3 showed higher improvement (B). In contrast, both in $4 \mathrm{kHz}(\mathrm{C})$ and $8 \mathrm{kHz}(\mathrm{D})$, Chavda type 2 showed higher improvement. Average of $0.5,1,2$ and $4 \mathrm{kHz}$ was compared and revealed high improvement in Chavda type 1 (E). In Siegel's criteria (F), proportion of Chavda type 1 was small in no improvement group. Nevertheless, all of these comparisons among Chavda types failed to reveal statistical significance (see the results for detailed statistics). The number in center of each bar means each mean hearing threshold improvement (dB HL). Error bar indicates standard deviation.

A

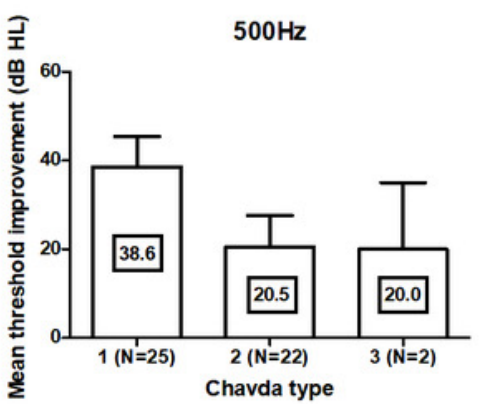

D

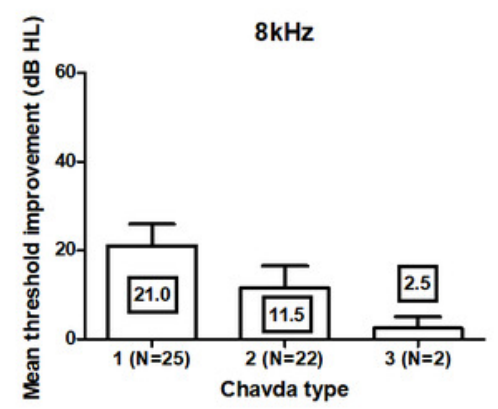

B

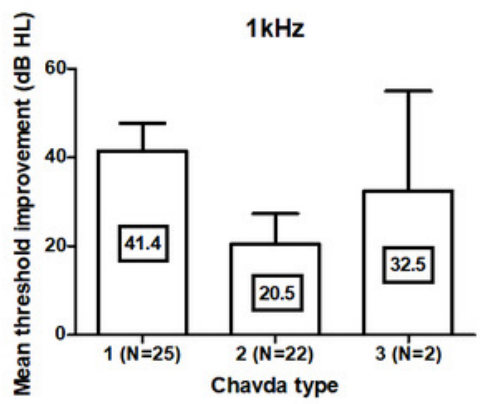

$\mathbf{E}$

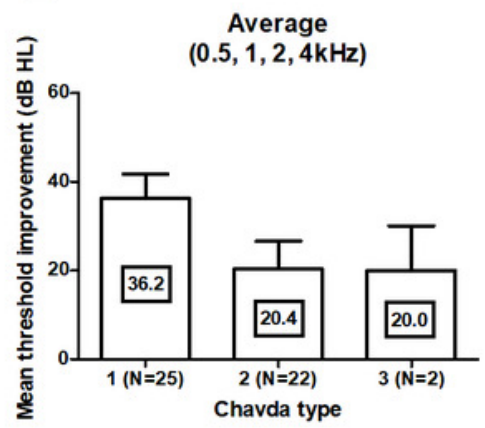

C

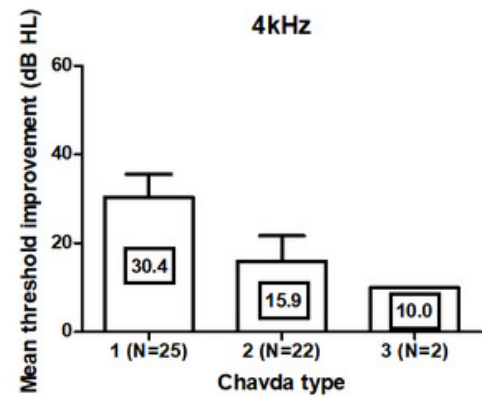

$\mathbf{F}$

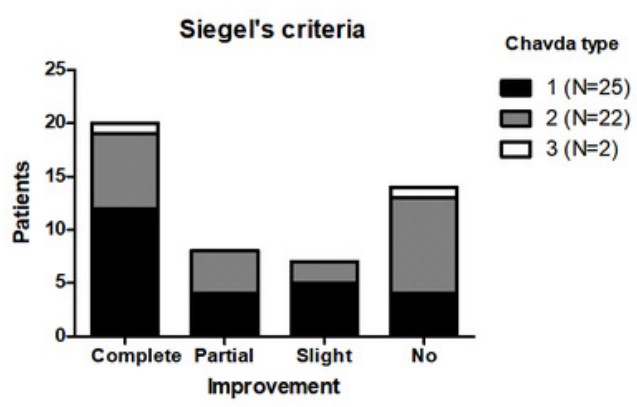




\section{Figure 4}

Hearing threshold improvement across different Gorrie types

At $500 \mathrm{~Hz}(\mathrm{~A})$ and $1 \mathrm{kHz}(\mathrm{B})$, Gorrie type 2 and 3 showed higher threshold improvement. At $4 \mathrm{kHz}$ (C) Gorrie type 4 showed least improvement of hearing. At $8 \mathrm{kHz}(\mathrm{D})$, threshold improvement across Gorrie types were similar. Average of $0.5,1,2$ and $4 \mathrm{kHz}$ was compared and showed similar Gorrie type distribution to $500 \mathrm{~Hz}$ and $1 \mathrm{kHz}$ (E). In Siegel's criteria (F), proportion of Gorrie types was not different among groups. Nevertheless, all of these comparisons among Gorrie types failed to reveal statistical significance (see the results for detailed statistics). The number in center of each bar means each mean hearing threshold improvement ( $\mathrm{dB} \mathrm{HL}$ ). Error bar indicates standard deviation.

A

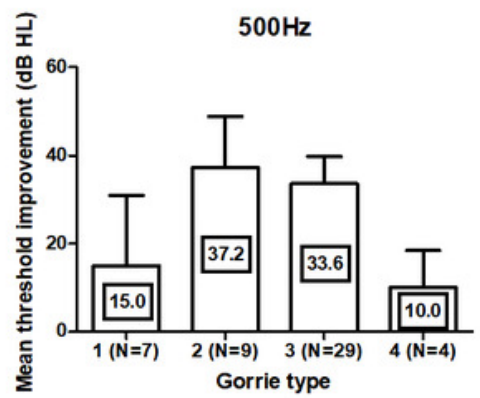

D

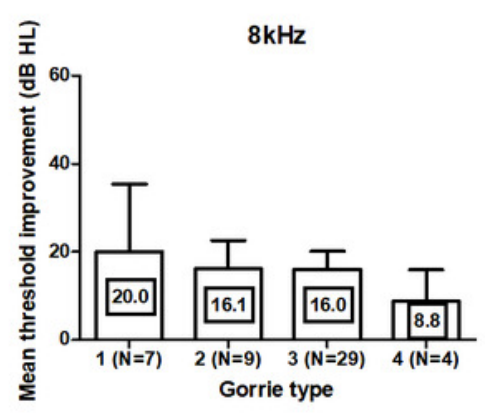

B

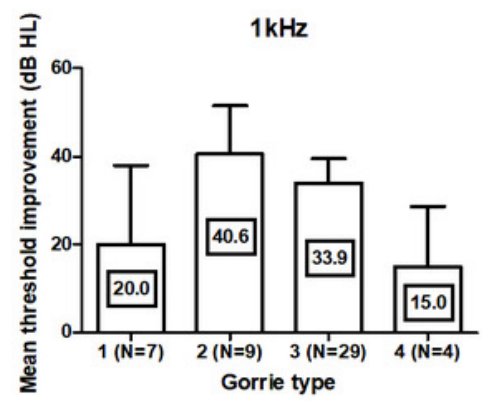

E

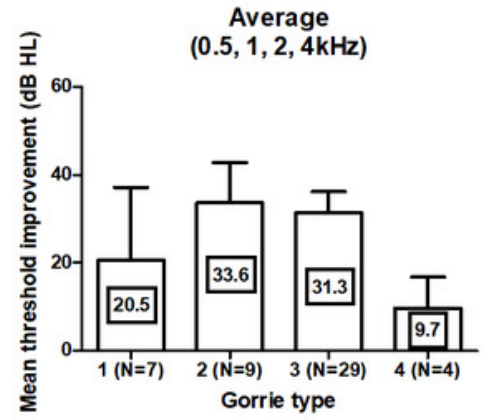

C

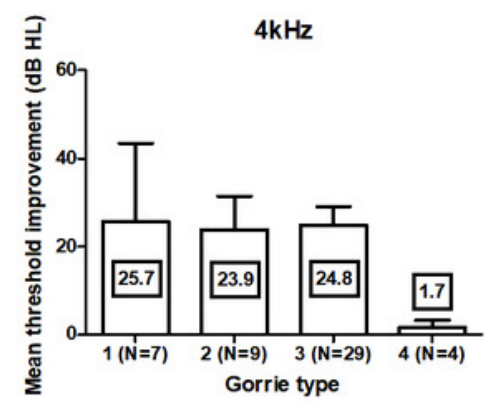

F

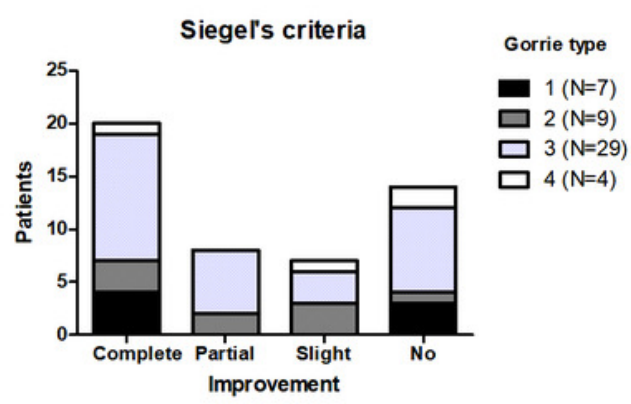

